\title{
Dynamic changes of CSF STREM2 in preclinical Alzheimer's disease: the CABLE study
}

Ling-Zhi Ma ${ }^{1+}$, Lan Tan ${ }^{1 \dagger}$, Yan-Lin Bi ${ }^{2 \dagger}$, Xue-Ning Shen ${ }^{3}$, Wei Xu' ${ }^{1}$ Ya-Hui Ma ${ }^{1}$, Hong-Qi $\mathrm{Li}^{3}$, Qiang Dong ${ }^{3}$ and Jin-Tai $Y u^{3^{*}}$

\begin{abstract}
Background: Loss of function of triggering receptor expressed on myeloid cell 2 (TREM2), a key receptor selectively expressed by microglia in the brain, contributes to the development of Alzheimer's disease (AD). Whether TREM2 levels are pathologically altered during the preclinical phase, and whether cerebrospinal fluid (CSF) soluble TREM2 protein (sTREM2) has a relationship with major pathological processes including $A \beta$ and tau deposition are still unclear.
\end{abstract}

Methods: According to the NIA-AA criteria, 659 cognitively normal participants from the Chinese Alzheimer's Biomarker and LifestylE (CABLE) cohort were divided into four groups, stage 0 (normal $A \beta_{1-42}$, T-tau and P-tau), stage 1 (low $A \beta_{1-42}$, normal T-tau and P-tau), stage 2 (low $A \beta_{1-42}$ and high T-tau or P-tau), and suspected non-AD pathology (SNAP) (normal $A \beta_{1-42}$ and high T-tau or P-tau), to examine changes of CSF sTREM2 in the preclinical AD. Biomarker cut-off was based on the assumption that one-third of adults with normal cognition have AD pathology.

Results: The level of CSF STREM2 in the stage 1 decreased compared with the stage $0(P<0.001)$, and then increased in the stage $2(P=0.008)$. SNAP individuals also had significantly increased CSF STREM2 $(P<0.001)$. Results of multiple linear regressions also showed positive correlations of CSF STREM2 with $A \beta_{1-42}(\beta=0.192$, $P<0.001)$, T-tau $(\beta=0.215, P<0.001)$ and $P$-tau $(\beta=0.123, P<0.001)$.

Conclusion: CSF STREM2 levels are dynamic in preclinical AD. A $\beta$ pathology is associated with a decrease in CSF STREM 2 in the absence of tau deposition and neurodegeneration. However, tau pathology and neurodegeneration are associated with an increase in CSF sTREM2.

Keywords: Alzheimer's disease, sTREM2, Inflammation, Biomarkers, Neurodegeneration

\footnotetext{
* Correspondence: jintai_yu@fudan.edu.cn

'Ling-Zhi Ma, Lan Tan and Yan-Lin Bi contributed equally to this work.

${ }^{3}$ Department of Neurology and Institute of Neurology, WHO Collaborating

Center for Research and Training in Neurosciences, Huashan Hospital,

Shanghai Medical College, Fudan University, 12th Wulumuqi Zhong Road,

Shanghai 200040, China

Full list of author information is available at the end of the article
}

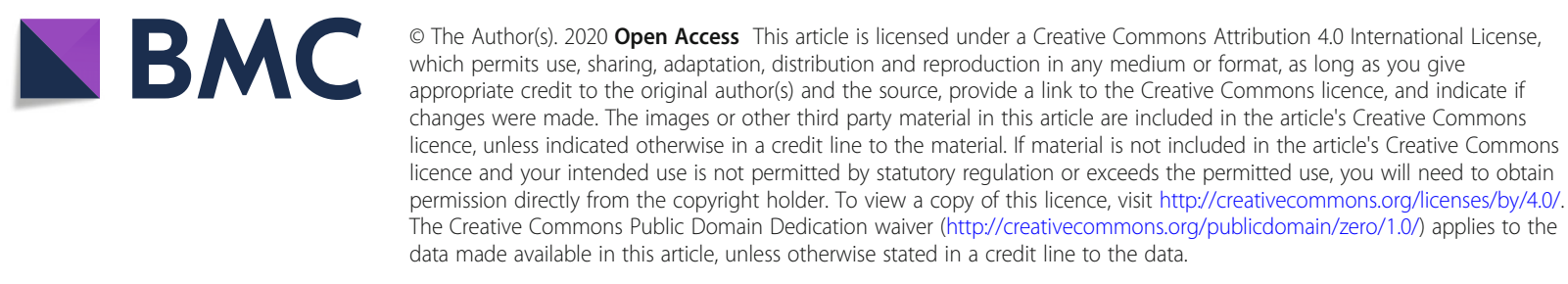




\section{Background}

Recent neuropathologic, epidemiologic and genetic studies have demonstrated that alterations of the innate immune system play a crucial role in the development of Alzheimer's disease (AD) [1, 2]. Triggering receptor expressed on myeloid cell 2 (TREM2) is expressed abundantly by microglia in the central nervous system (CNS) and involved in key functions of microglia, including phagocytosis, cytokine release, lipid sensing, microglia proliferation, and migration [3-5]. Rare variants in the gene encoding the triggering receptor expressed on TREM2 are associated with increased risk of AD and other neurodegenerative diseases [6, 7]. TREM2 undergoes ectodomain shedding by ADAM proteases, and a soluble fragment, namely soluble TREM2 (sTREM2), is released into the extracellular space $[4,8]$. sTREM2 can be detected in cerebrospinal fluid (CSF) by a method based on high sensitivity enzyme-linked immunosorbent assay (ELISA) [4, 9]. TREM2 is associated with AD and neurodegeneration, which leads us to hypothesize that CSF sTREM2 may be a marker of microglial function, and microglial response to amyloid $\beta(A \beta)$, tau pathology and neurodegeneration.

Previous studies of patients with late-onset AD reported that CSF sTREM2 increased in a disease-stagedependent manner and peaked in the early symptom phase $[10,11]$. In studies of autosomal dominant mutation carriers, CSF STREM2 increased 5 years prior to the onset of symptom onset (EYO), however, after about 5 to 10 years of $A \beta$ pathology development [12]. Taken together, these studies suggest a complex link between CSF sTREM2 and disease progression, in which CSF sTREM2 dynamically changes with AD progression and peaks between the later asymptomatic and earlier symptomatic phases. However, these studies were only limited in clinical stage. Increased microglial activation and neuroinflammation frequently accompany the early development of $A \beta$ and tau pathology [10]. Since TREM2 is a key protein involved in the activation of microglia, a question arises as to whether the level of sTREM2 changes pathologically during the preclinical phase, which we addressed in the current study.

Therefore, we used the biomarker-based hypothetical staging model to investigate CSF STREM2 levels in different groups of cognitive normal divided by AD biomarkers $[13,14]$. This model proposes that the asymptomatic phase of $\mathrm{AD}$ can be divided into 3 successive stages according to aggregated $A \beta\left(A \beta_{1-42}\right)$, aggregated tau (phosphorylated tau, P-tau) and neurodegeneration (total tau, T-tau). We also tested whether CSF sTREM2 levels were associated with the core AD CSF biomarkers $A \beta_{1-42}, A \beta_{1-}$ ${ }_{40}$, T-tau and P-tau in healthy controls $(\mathrm{HC})$ and preclinical AD groups [15]. We limited the study to cognitively normal individuals. Because the preclinical phase of $\mathrm{AD}$ is the focus of clinical trials, as it is speculated that intervention may be the most successful when treated before the initial symptom phase known as mild cognitive impairment (MCI) [16, 17].

\section{Methods \\ CABLE database}

Chinese Alzheimer's Biomarker and LifestylE (CABLE) study is an ongoing large-scale cohort study initiated in 2017, mainly focusing on Alzheimer's disease risk factors and biomarkers in the northern Chinese Han population. CABLE is aimed to determine the genetic and environmental modifiers of $\mathrm{AD}$ biomarkers and to identify lifestyle factors that may affect the risk of $\mathrm{AD}$ in the non-demented northern Chinese Han population, thus providing a basis for disease prevention and early diagnosis. In conjunction with the investigation, oral informed consent for future use of their CSF and blood samples for research purposes was obtained. All patients were later instructed to withdraw their permission if they changed their minds. The design of the study was approved by the Institutional Review Board of Qingdao Municipal Hospital, and the study procedure was conducted in accordance with the Declaration of Helsinki.

\section{Participants}

All enrolled participants in the CABLE were Han Chinese aged between 40 to 90 years old. The exclusion criteria include: (1) central nervous system infection, head trauma, multiple sclerosis, neurodegenerative diseases other than $\mathrm{AD}$ (e.g., epilepsy, Parkinson's Disease), or other major neurological disorders; (2) major psychological disorders; (3) severe systemic diseases (e.g., malignant tumors) that may affect CSF or blood levels of AD biomarkers including $A \beta$ and tau; (4) family history of genetic diseases. All participants received clinical and neuropsychological assessments, biochemical testing, as well as blood and CSF sample collection. Comprehensive questionnaire, electronic medical record system, and a laboratory inspection management system were used to collect demographic information, $\mathrm{AD}$ risk factor profile and medical history. The diagnostic criteria of preclinical AD was defined according to the 2011 National Institute on Aging-Alzheimer's Association (NIA-AA) workgroup reports, that is, normal cognition but abnormal AD biomarkers [14]. We first screened cognitive state of participants using the China Modified Mini-Mental State Examination (CM-MMSE) and Montreal Cognitive Assessment (MoCA). All diagnoses were evaluated by two medical doctors with extensive experience in cognitive disorders through intact performance on neuropsychological tests combined with CSF biomarkers and MRI examinations.

A total of 1000 cognitively normal participants from CABLE had available information on covariates. Sixty- 
nine participants were excluded who had no information about CM-MMSE, and 215 participants without available CSF sTREM2 data were excluded. Fifty-seven participants without CSF biomarkers and those had data outside the standard deviation (SD) of four times were removed. Finally, 659 participants were included in this cross-sectional analysis. Frequency histogram of age distribution of populations was shown in Figure S1.

\section{CSF core biomarkers and CSF sTREM2 measurements}

Collection and storage of fasting lumbar CSF samples and blood samples were performed at Qingdao Municipal Hospital. CSF samples were processed immediately within $2 \mathrm{~h}$ after standard lumbar puncture. Each sample was centrifuged at $2000 \times \mathrm{g}$ for $10 \mathrm{~min}$, and CSF samples were separated and stored in an enzyme-free EP (Eppendorf) tube (AXYGEN; PCR-02-C) at $-80^{\circ} \mathrm{C}$ for further use in the subsequent steps of this study. The samples were subjected to a maximum of two freeze-thaw cycles. The DNA in the blood samples extracted using the QIAamp DNA Blood Mini Kit (250) was separated and stored in an enzyme-free EP tube at $-80^{\circ} \mathrm{C}$ until the apolipoprotein $\mathrm{E}(A P O E)$ genotyping in this study. Two specific loci related to $A P O E$ status (rs7412 and rs429358) were selected for genotyping with restriction fragment length polymorphism (RFLP) technology.

CSF sTREM2 and core biomarkers were measured by ELISA using the microplate reader (Thermo Scientific Multiskan MK3). CSF sTREM2 measurements were done with ELISA kits (Human TREM2 SimpleStep ELISA kit; Abcam, no. Ab224881) and CSF core biomarkers measurements were done with other ELISA kits (INNOTEST; FUJIREBIO). All ELISA measurements were performed by experienced technicians in strict accordance with the manufacturer's instructions. They were blinded to the clinical information. The samples and standards were measured in duplicate, and the means of duplicates were used for the statistical analyses. All the antibodies and plates were from a single lot to exclude variability between batches. Moreover, the within-batch $\mathrm{CV}$ was $<5 \%$ and the inter-batch $\mathrm{CV}$ was $<15 \%$. Furthermore, Figure S2 shows that CSF $A \beta_{1-42}$ was reduced with $A P O E \& 4$ when stratifying the whole cohort for $A P O E$ genotype and that it decreased with age. It indicated that raw data is sound with no technical problems [18].

\section{Statistical analysis}

The scheme comprises 3 biomarkers: aggregated $A \beta\left(A \beta_{1-}\right.$ $\left.{ }_{42}\right)$, aggregated $\mathrm{AD}$-tau (P-tau) and neurodegeneration ( $\mathrm{T}$ tau). And each biomarker is binarized based on whether they are normal or abnormal. The observation that approximately one-third of cognitively normal older adults have $\mathrm{AD}$ pathology in their brains has been approved by previous amyloid imaging [18-20] and neuropathological studies [21, 22]. Similar distributions were found in studies of Asian populations [23-25]. Therefore, the cutoff values to define abnormal CSF core biomarkers were $<115.1 \mathrm{pg} / \mathrm{ml}$ (lower onethird) for $\mathrm{A} \beta_{1-42},>40.05 \mathrm{pg} / \mathrm{ml}$ (upper one-third) for P-tau, and $>187.32 \mathrm{pg} / \mathrm{ml}$ (upper one-third) for T-tau, respectively Aggregated tau and neurodegeneration groups were merged to reduce the number of groups to be compared, which resulted in four different biomarker group combinations, including stage 0 , stage 1 , stage 2 , and suspected non- $A D$ pathology (SNAP). Individuals with normal measures of $\mathrm{A} \beta_{1-42}$, P-tau and T-tau are classified as stage 0 ; individuals with abnormal $\mathrm{A} \beta_{1-42}$ but no abnormal P-tau or T-tau are classified as stage 1 ; during stage 2 , abnormal $A \beta_{1-42}$, and abnormal P-tau or T-tau are evident. Individuals with evidence of aggregated tau or neurodegeneration but with normal levels of amyloid are classified as having SNAP [26]. Participants were also classified into $\mathrm{HC}$ (stage 0 ) and preclinical $\mathrm{AD}$ (stage 1 and stage 2) according to the Alzheimer's continuum category [14, 27, 28].

CSF sTREM2 didn't follow a normal distribution as assessed by Kolmogorov-Smirnov test $(P=0.200)$ and visual inspection of the $\mathrm{Q}-\mathrm{Q}$ plot (Figure S3). Therefore, they were log-transformed to obtain a normal distribution. All the statistical analyses described in this study are performed on the log10-transformed values. We performed the analysis after excluding outliers (defined as 4 SD below or above the group mean) in order to exclude the influence of extreme values. This step removed 3 participants. Tests of intergroup differences were performed using the chi-square analysis for frequencies or one-way analysis of variance and post- hoc analysis for continuous measures. We used the Student $t$-test to explore whether CSF sTREM2 differs with age, gender and $A P O E \& 4$ carrier status. The APOE $\& 4$ carrier status was coded as 0,1 , and 2 , respectively. To test the differences in CSF sTREM2 across biomarker profiles in the biomarker framework, we applied a one-way ANCOVA followed by Bonferroni post hoc analyses. We also studied the associations between CSF sTREM2 and the CSF core biomarkers for $\mathrm{AD}$, in $\mathrm{HC}$ (stage 0 ) and preclinical $\mathrm{AD}$ (stage 1 and stage 2) groups, with a multiple linear regression adjusted for age, gender, educational level, and $A P O E \& 4$ carrier status. The analyses were performed in the total sample and then in subgroups stratified by age, gender and $A P O E \varepsilon 4$ carrier status.

Statistical significance was defined as $p<0.05$ for all analyses. Statistical analyses were performed using $\mathrm{R}$ (version 3.5.1) and IBM SPSS Statistics 23.

\section{Results}

Basic characteristics and intergroup comparisons

We studied a total of 659 participants (242 stage 0, 148 stage 1, 70 stage 2, and 199 SNAP) of the CABLE study. 
Table 1 Characteristics of participants by biomarker framework

\begin{tabular}{|c|c|c|c|c|c|}
\hline Characteristics & Stage 0 & Stage 1 & Stage 2 & SNAP & $P$ \\
\hline $\bar{n}$ & 242 & 148 & 70 & 199 & \\
\hline Age (years) & $60.81 \pm 9.95$ & $60.39 \pm 10.41$ & $64.19 \pm 11.09$ & $64.59 \pm 10.28$ & $<0.001$ \\
\hline Gender (F/M) & $96 / 146$ & $63 / 85$ & $31 / 39$ & $73 / 126$ & 1.000 \\
\hline Education (years) & $9.96 \pm 4.39$ & $10.17 \pm 4.20$ & $9.24 \pm 4.56$ & $9.32 \pm 4.37$ & 0.188 \\
\hline APOE \&4 carriers (\%) & $38(15.70)$ & $22(14.86)$ & $18(25.71)$ & $27(13.57)$ & 1.000 \\
\hline CM-MMSE score & $27.94 \pm 2.25$ & $27.92 \pm 1.99$ & $27.41 \pm 2.18$ & $27.61 \pm 2.06$ & 0.155 \\
\hline \multicolumn{6}{|l|}{ CSF biomarker } \\
\hline$A \beta_{1-42}(p g / m l)$ & $170.41 \pm 63.10$ & $103.31 \pm 7.26$ & $102.95 \pm 6.31$ & $224.78 \pm 139.64$ & $<0.001$ \\
\hline$A \beta_{1-40}(p g / m l)$ & $5669.91 \pm 1855.05$ & $4567.68 \pm 1906.91$ & $7379.50 \pm 2545.57$ & $8493.87 \pm 2982.58$ & $<0.001$ \\
\hline T-tau (pg/ml) & $135.72 \pm 25.95$ & $124.98 \pm 2.55$ & $262.85 \pm 104.36$ & $241.30 \pm 88.10$ & $<0.001$ \\
\hline P-tau (pg/ml) & $33.18 \pm 3.85$ & $31.49 \pm 3.87$ & $46.13 \pm 10.84$ & $46.99 \pm 11.37$ & $<0.001$ \\
\hline$A \beta_{1-42} / A \beta_{1-40}$ (Median, IQR) & $0.029(0.024-0.037)$ & $0.024(0.018-0.032)$ & $0.014(0.011-0.018)$ & $0.025(0.18-0.031)$ & $<0.001$ \\
\hline T-tau/A $\beta_{1-42}$ (Median, IQR) & $0.84(0.69-1.03)$ & $1.20(1.02-1.36)$ & $2.22(1.85-3.02)$ & $1.13(0.90-1.58)$ & $<0.001$ \\
\hline P-tau/A $\beta_{1-42}$ (Median, IQR) & $0.21(0.17-0.25)$ & $0.31(0.28-0.33)$ & $0.42(0.39-0.51)$ & $0.24(0.19-0.31)$ & $<0.001$ \\
\hline sTREM2 (pg/ml) & $18,000.57 \pm 6344.52$ & $15,121.87 \pm 6396.85$ & $18,282.53 \pm 7538.97$ & $20,814.16 \pm 6105.39$ & $<0.001$ \\
\hline
\end{tabular}

Categorical variables are reported as numbers and percentages; continuous variables are reported as means \pm SDs

Abbreviations: SNAP Suspected non-Alzheimer disease pathology, SD Standard deviations, F Female, M Male, APOE Apolipoprotein E, CM-MMSE China Modified Mini-Mental State Examination, CSF Cerebrospinal fluid; $A \beta_{1-42}$, amyloid- $\beta_{1-42} ; A \beta_{1-40}$, amyloid- $\beta_{1-40}$; T-tau, total tau; P-tau, phosphorylated tau; sTREM2, soluble TREM2

The demographical and clinical characteristics are described in Table 1. The resulting proportion of individuals in the hypothetical groups was comparable to that reported in the literature [26]. The study population had a female proportion of $40 \%$, an age ranges from 40 to 90 years old (mean $\pm \mathrm{SD}=62.2 \pm 10.4$ ), education of $9.7 \pm 4.4$ years, and an $A P O E \varepsilon 4$ positive percentage of $16 \%$. As for cognitive performance, the study population had CMMMSE of $27.8 \pm 2.1$ scores. There were no differences between the four groups in terms of gender, educational level, CM-MMSE score. However, individuals in stage 2 group were older and had lower cognitive composite scores than the HC group.
Since age is the main risk factor for $\mathrm{AD}$, we questioned whether CSF sTREM2 levels are related to normal aging. We found sTREM2 levels to increase with age, as demonstrated by a significant positive correlation $(\beta=0.004$, $P<0.001$ ) (Figure S4). The results indicated that CSF sTREM2 did differ significantly in different age subgroups (middle-age: $17511.5 \pm 6615.2 \mathrm{pg} / \mathrm{ml}, \quad n=376$; elder: $\quad 19193.1 \pm 6769.5 \mathrm{pg} / \mathrm{ml}, \quad n=283 ; \quad P=0.005)$ (Fig. 1a). As expected, CSF sTREM2 concentrations were not affected by gender (male $=18,190.3 \pm 6815.4 \mathrm{pg} / \mathrm{ml}$, $n=263$; female $=18,298.9 \pm 6608.0 \mathrm{pg} / \mathrm{ml}, n=396 ; P=$ $0.576)$ (Fig. 1b), and $A P O E$ carrier status $(A P O E \varepsilon 4$ negative $=18,287.0 \pm 6704.0 \mathrm{pg} / \mathrm{ml}, n=554 ; A P O E \varepsilon 3 / \varepsilon 4=17$,
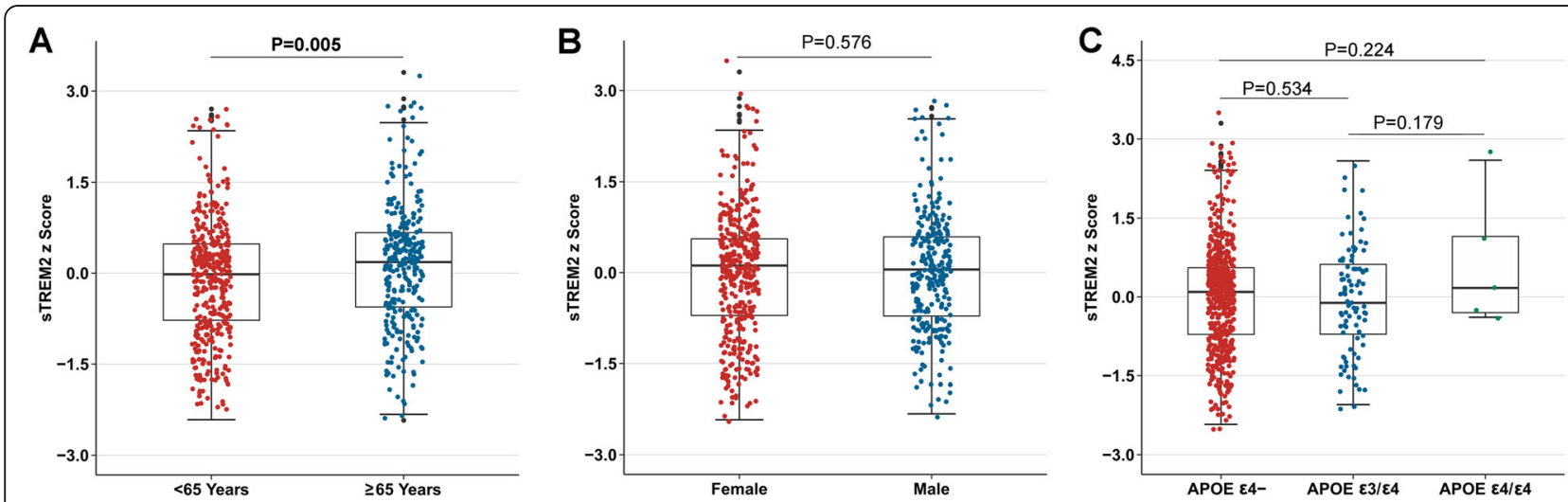

Fig. 1 CSF STREM2 levels are associated with age (a) and are independent of gender (b) and APOE carrier status (c). P-values were assessed by student's t test. Abbreviations: CSF, cerebrospinal fluid; sTREM2, soluble TREM2; APOE, apolipoprotein E 
$793.5 \pm 6837.8 \mathrm{pg} / \mathrm{ml}, n=100 ; A P O E \varepsilon 4 / \varepsilon 4=22,613.1 \pm$ $8441.9 \mathrm{pg} / \mathrm{ml}, n=5$ ) (Fig. 1c).

\section{Difference in CSF sTREM2 level between biomarker categories}

A representative standard curve for the CSF sTREM2 ELISA is shown in the Figure S5. A standard curve was created by plotting the average blank control subtracted absorbance value for each standard concentration (y-axis) against the target protein concentration ( $\mathrm{x}$-axis) of the standard. A four-parameter curve fit (4PL) was chosen to draw the best smooth curve through these points to construct the standard curve. To assess the associations of change in CSF sTREM2 with $A \beta$ deposition and the downstream processes of the amyloid cascade (ie, tau pathology and neurodegeneration), we applied the biomarker classification framework and compared the differences in sTREM2 levels between the four stages, namely stage $0(n=242)$, stage $1(n=148)$, stage $2(n=70)$, and SNAP $(n=199)$. The results of one-way ANCOVA showed significant differences in CSF sTREM2 levels between the four groups. The results of the comparison between groups showed that the stage 1 group had the significantly lowest CSF sTREM2 compared to other groups (Fig. 2$)$. Both the stage $2(P=0.008)$ and the SNAP $(P<0.001)$ groups had significantly increased CSF sTREM2 concentrations compared to the stage 1 group (Fig. 2). It can be inferred that the concentration of CSF sTREM2 decreases with $A \beta$ in the pathological phase of amyloid, and then increases with T-tau or P-tau in

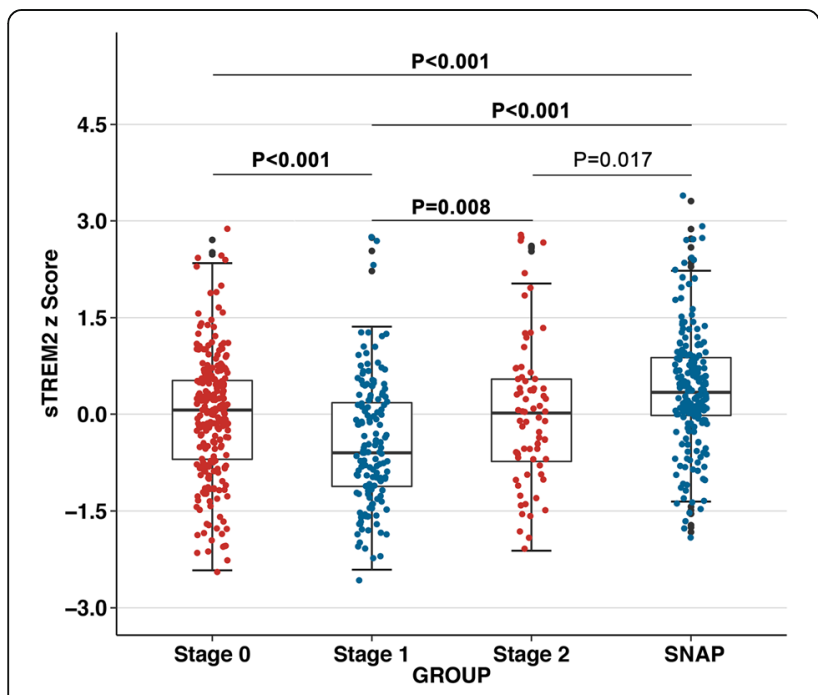

Fig. 2 CSF STREM2 in the biomarker classification. Scatter plots depicting the levels of CSF STREM2 for each of the four biomarker profiles (stage 0 , stage 1, stage 2, and SNAP). P-values were assessed by a one-way ANCOVA, and significant $P$ values after Bonferroni corrected post hoc pairwise comparisons are marked. Abbreviations: CSF, cerebrospinal fluid; sTREM2, soluble TREM2; SNAP, suspected non-Alzheimer disease pathology downstream tau pathology and neurodegeneration. Changes in CSF sTREM2 occurred after alterations in markers for brain amyloidosis and neuronal injury.

Because the results suggest that age is an important influencing factor for CSF sTREM2, we conducted another ANCOVA controlling for age, and obtained the same results as follows. The stage 1 group had significantly lowest CSF sTREM2 compared to other groups $(P<0.001)$. Both the stage $2(P=0.021)$ and the SNAP $(P<0.001)$ groups had higher CSF sTREM2 concentrations compared to the stage 1 group. We also repeated the same analyses in groups divided by participants' $A \beta$ pathology $\left(\mathrm{A} \beta_{1-42}\right)$ and tau pathological status (P-tau) or in groups divided by participants' $A \beta$ pathology $\left(A \beta_{1-42}\right)$ and neurodegenerative state ( $\mathrm{T}$-tau). The results are shown in Figure S6 and Figure S7, which are similar to the results in the former classification system.

\section{CSF sTREM2 levels in asymptomatic cognitively normal SNAPs}

The SNAPs refer to cognitively normal individuals with abnormal tau pathology/ neurodegeneration biomarkers without the presence of significant amyloidosis [26]. We compared the SNAPs with the rest of the asymptomatic cognitively normal individuals, including $\mathrm{HC}$ (stage 0 ) and preclinical AD (stage 1and 2). We found that CSF sTREM2 levels differed among groups and particularly increased in the SNAP group compared to the HC $(P<0.001)$ and the preclinical $\mathrm{AD}$ (stage $1, P<0.001$; stage $2, P=0.017$ ) groups (Fig. 2). The results remained after controlling for age. These findings indicate that an increased CSF sTREM2 level is a response to tau pathology or neuronal injury (measured by CSF P-tau or T-tau levels), independent of amyloidosis.

\section{Relations between CSF sTREM2 and AD core biomarkers}

The associations between CSF sTREM2 and core CSF markers of $\mathrm{AD}$ were tested in linear regression models adjusted for age, gender, educational level, and $A P O E \varepsilon 4$ carrier status. Those individuals that fall in the category of SNAP were excluded from this analysis. In the whole sample of subjects $(n=659)$, increased CSF sTREM2 was associated with higher levels of $A \beta_{1-42}(\beta=0.192$, $P<0.001)$ (Fig. 3a) and $A \beta_{1-40}(\beta=0.318, P<0.001)$ (Fig. 3d), as well as higher levels of T-tau $(\beta=0.215, P<$ 0.001) (Fig. 3g) and P-tau ( $\beta=0.123, P<0.001)$ (Fig. 3f). The results of the subgroup analysis showed that the relationship between sTREM2 and CSF biomarkers remained unchanged regardless of age, gender, and APOE \&4 carrier status (Table S2). There was a positive association between CSF sTREM2 and T-tau or P-tau in HC $(n=242)$ and preclinical AD groups $(n=218)$ (Fig. $3 \mathrm{~h}-\mathrm{i}$, Fig. 3k-l), whereas no significant association was found between CSF sTREM2 and $A \beta_{1-42}$ in HC group $(\beta=0.056, P=0.198$ ) (Fig. 3b). We then examined ratios 

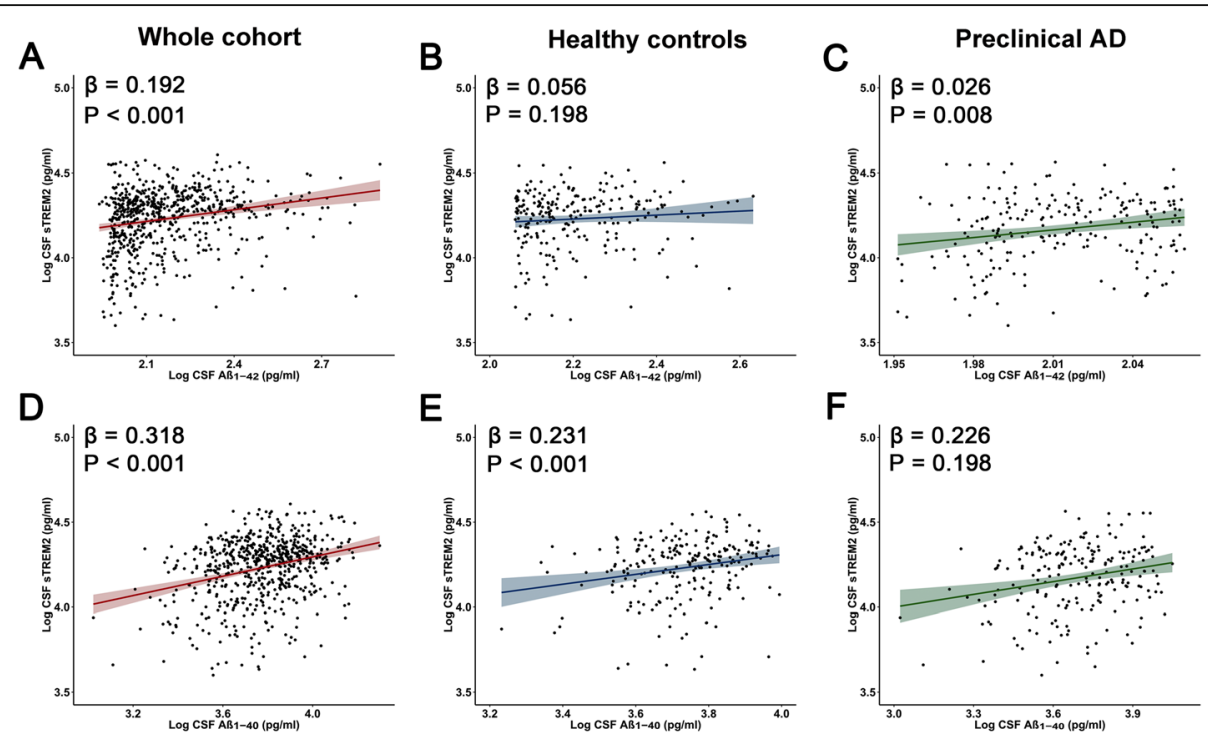

F
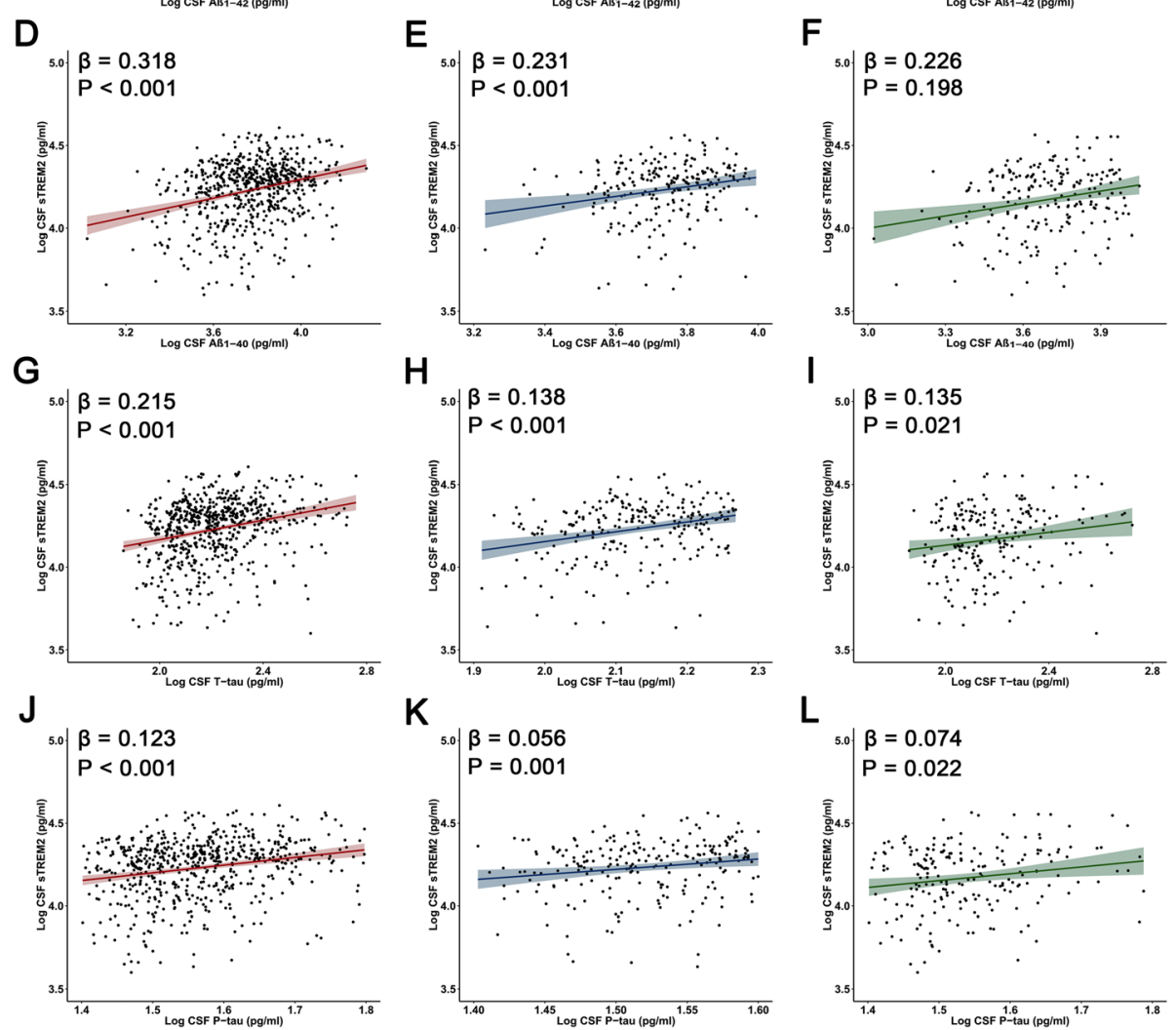

Fig. 3 Associations of CSF sTREM2 and CSF core biomarkers. Scatter plots represent the associations of CSF sTREM2 with CSF core biomarkers: A $\beta_{1-42}$, $A \beta_{1-40}$, T-tau, and P-tau in three different groups (whole cohort, healthy controls, and preclinical AD). The normalized regression coefficients ( $\beta$ ) and $P$ values computed by multiple linear regression after adjustment for age, gender, educational level, and APOE $\varepsilon 4$ carrier status are shown. Abbreviations: CSF, cerebrospinal fluid; sTREM2, soluble TREM2; $A \beta_{1-42}$, amyloid- $\beta_{1-42} ; A \beta_{1-40}$, amyloid- $\beta_{1-40}$; T-tau, total Tau; P-tau, phosphorylated Tau; $A D, A P O E$, apolipoprotein E

of CSF amyloid and tau biomarkers, and found no associations of CSF sTREM2 with CSF $A \beta_{1-42} / A \beta_{1-40}$, Ttau $/ A \beta_{1-42}$ and $P$-tau/A $\beta_{1-42}$ (Table S1). Outliers were excluded in our analyses, but we obtained similar results when those were included. These finding indicate that higher CSF sTREM2 correlates with higher levels of markers for neuronal injury and tau pathology, suggesting an early response of TREM2 to the first symptoms of neurodegeneration.

\section{Discussion}

In the present study, we combined biomarker-based classification with clinical stages to assess changes in the microglial-activity marker CSF sTREM2 within the early phases of $\mathrm{AD}$. The application of this classification system enabled us to explore the associations of microglial inflammatory response with $\mathrm{AD}$ pathophysiology (including $\mathrm{A} \beta$ pathology, tau pathology, and neurodegeneration). Our study extends previous evidence by showing that CSF sTREM2 levels did change dynamically with pathological processes. The simple A $\beta$ pathology (defined as low CSF $\left.\mathrm{A} \beta_{1-42}\right)$ is associated with a decrease in CSF sTREM2, while tau pathology or neurodegeneration is associated with elevated CSF sTREM2. This seems to confirm the potential role of microglial inflammatory response in the pathogenesis of $\mathrm{AD}$. Our data provide support for this hypothesis: $A \beta$ deposition occurs independently of the inflammatory process, but the type and extent of the 
inflammatory response to $A \beta$ deposition in the brain may trigger or affect subsequent neurodegeneration [29]. Another piece of evidence is that immunotherapy against amyloid can reduce downstream neurodegeneration, a process that may be mediated by changes in microglial activation [29].

Consistent with previous studies, the findings of the present study indicate that higher CSF sTREM2 concentrations are linked to tau pathology or neurodegeneration (defined as elevated CSF P-tau or T-tau) even in the absence of $A \beta$ pathology, as indicated by the differences between HC and SNAP individuals [12, 30]. This suggests that an increased inflammatory response and involvement of reactive microgliosis are related to neurodegeneration. Without $A \beta$ pathology, increased sTREM2 seems to be a consequence rather than a cause of neurodegeneration, which is in line with reports that the TREM2 receptor mediated the phagocytosis of apoptotic neurons by microglia [31]. In addition, the SNAP category is noteworthy because it is the heterogeneous group most likely to exhibit non-AD-related neurodegeneration. Future studies should address changes in CSF sTREM2 in neurodegenerative diseases other than AD.

Exploring relations between CSF biomarkers may provide better pathogenic understanding by hinting to simultaneously ongoing processes in the brain. Our results are consistent with previous research studies [10-12, 32]. The level of CSF sTREM2 was positively correlated with the core CSF biomarkers $A \beta_{1-42}, A \beta_{1-40}$, T-tau and $\mathrm{P}$-tau on the entire data set and in preclinical AD, which further suggests an early involvement of reactive microgliosis. In $\mathrm{HC}$ subjects, the correlation with $\mathrm{A} \beta_{1-42}$ disappeared, while the correlations of sTREM2 with T-tau and P-tau remained. These findings suggest that changes in CSF sTREM2 may indeed be associated with neuronal injury. It is also suggested that the increase in CSF sTREM2 during normal aging may be a protective response to mild neuronal injury. Furthermore, we showed that sTREM2 did not seem to depend on the APOE $\varepsilon 4$ genotype, as there was no difference in CSF sTREM2 concentration between $A P O E \& 4$ carriers and noncarriers; sTREM2 also did not depend on gender because there was no difference in CSF sTREM2 concentration between males and females.

The underlying mechanism of dynamic changes in CSF sTREM2 remains to be investigated throughout the disease. The mechanism underlying the reduction of CSF STREM2 in the early stage of $A \beta$ pathology is still unclear. One explanation is that microglia initially form a barrier around the plaque, and sTREM2 released by microglia remains in the plaque until the barrier fails, followed by neural injury [33, 34]. Another explanation is that individuals with low function of TREM2 may have early amyloidogenic acceleration, so the level of
sTREM2 in the preclinical AD stage 1 is lower [35]. Increased sTREM2 is caused by microglial activation, which is evidenced by increased TREM2 expression both in elderly $\mathrm{AD}$ mouse models and in human $\mathrm{AD}$ brains [36, 37]. In addition, detailed transcriptomics studies investigated microglia in $\mathrm{AD}$ and neurodegenerative mouse models, suggesting that TREM2 is up-regulated in disease-associated microglia (DAM) [38-40]. We found positive correlations of CSF sTREM2 with T-tau and P-tau in the control group and preclinical group, which was consistent with previous studies $[10,12]$. Since neuroinflammation is a feature of aging, [41, 42], it cannot be ruled out that correlation of CSF sTREM2 levels with $\mathrm{T}$-tau/P-tau in $\mathrm{HC}$ group could thus be an indirect effect of the age-dependent increase in T-tau/Ptau. Another possibility is that studies have suggested that the increase in CSF tau in AD is due to increased tau production (ie, synthesis and release) rather than decreased fractional clearance. Under normal physiological conditions, soluble tau and CSF tau in the brain are in equilibrium. However, mechanisms such as inflammation may increase the production or release of tau into the extracellular space, and over time, the concentration of soluble extracellular tau will increase [43]. CSF sTREM2 will be an attractive biomarker candidate to track AD progression and it can serve as a potential outcome parameter for future clinical trials of TREM2 and neuroinflammation.

The strength of the current study is a large sample of well-characterized subjects with biomarker evidence of $\mathrm{AD}$ pathology. We used an $\mathrm{AD}$ biomarker classification system in line with NIA-AA research diagnostic guidelines for $\mathrm{AD}$. This allowed us to study patients in the preclinical stages of AD. Previous studies mainly focused on $\mathrm{AD}$ patients, with a few participants in the preclinical phase, and had no biomarker classification [12, 44]. However, our study also has some limitations. First, we did not screen subjects for possible TREM2 mutations. However, the possibility of TREM2 mutations in current patient samples is highly unlikely to affect our results, as the prevalence of TREM2 mutations in the general population or AD patients is low [7, 45]. Second, this is a cross-sectional study that limits any conclusions about disease progression. Therefore, results should be replicated in subjects with longitudinal data to analyze whether CSF sTREM2 levels are associated with disease progression.

\section{Conclusion}

In conclusion, this study is based on an independent cohort. The results indicate that CSF sTREM2 is dynamic in preclinical $A D$. $A \beta$ pathology is associated with a decrease in CSF sTREM2 in the absence of tau deposition and neurodegeneration, whereas tau pathology and 
neurodegeneration is associated with an increase in CSF sTREM2. Future studies should use biomarkers to further categorize the preclinical AD stage and explore the biological mechanisms underlying the dynamic CSF sTREM2 changes.

\section{Supplementary information}

Supplementary information accompanies this paper at https://doi.org/10. 1186/s13024-020-00374-8.

Additional file 1: Figure S1. Histogram of age distribution of CABLE populations. Figure S2. Associations of age and the presence or absence of $A P O E \varepsilon 4$ for CSF measures of $A \beta_{1-42}$. Figure S3. The Quantile-Quantile plot of CSF sTREM2. Figure S4. Association of CSF sTREM2 and age. Figure S5. One of the standard curves for CSF sTREM2. Figure S6.CSF sTREM2 in groups defined only by CSF $A \beta_{1-42}$ and P-tau. Figure S7. CSF sTREM 2 in groups defined only by CSF $A \beta_{1-42}$ and T-tau. Table S1. Association of CSF sTREM2 and CSF core biomarkers. Table S2. Subgroup analyses of whole participants by age, gender, and APOE $\varepsilon 4$ carrier status.

\section{Abbreviations}

AD: Alzheimer's disease; TREM2: Triggering receptor expressed on myeloid cell 2; CNS: Central nervous system; sTREM2: Soluble TREM2;

CSF: Cerebrospinal fluid; ELISA: Enzyme-linked immunosorbent assay; $A \beta$ : Amyloid $\beta_{;} A \beta_{1-42}$ : Amyloid- $\beta_{1-42} ;$ P-tau: Phosphorylated tau; T-tau: Total tau; $\mathrm{HC}$ : Healthy control; $A \beta_{1-40}$ : Amyloid- $\beta_{1-40}$; MCl: Mild cognitive impairment; CABLE: Chinese Alzheimer's Biomarker and Lifestyle; NIAAA: Aging-Alzheimer's Association; CM-MMSE: China Modified Mini-Mental State Examination; SD: Standard deviation; APOE: Apolipoprotein E; RFLP: Restriction fragment length polymorphism; SNAP: Suspected non-AD pathology

\section{Acknowledgements}

The authors thank the colleagues who have made contributions to build the CABLE cohort. The authors also thank the subjects and their family for their cooperation in this study.

\section{Authors' contributions}

$L Z M, L T, Y L B$, and JTY did the manuscript preparation and drafting. LZM, LT, YLB, XW, XNS, YHM, and JTY did the clinical assessments and data acquisition. LT and JTY did the clinical diagnosis. LZM, LT, YLB, XW, XNS, HQL, YHM, QD, and JTY did the data analysis and interpretation. LT and JTY is responsible for the study conception and design. All authors have contributed to the manuscript revising and editing critically for important intellectual content and given final approval of the version and agreed to be accountable for all aspects of the work presented here. All authors read and approved the final manuscript.

\section{Funding}

This study was supported by grants from the National Key R\&D Program of China (2018YFC1314700), the National Natural Science Foundation of China (91849126), Shanghai Municipal Science and Technology Major Project (No.2018SHZDZX01) and ZHANGJIANG LAB, Tianqiao and Chrissy Chen Institute, and the State Key Laboratory of Neurobiology and Frontiers Center for Brain Science of Ministry of Education, Fudan University.

\section{Availability of data and materials}

The data that support the findings of this study are available from the corresponding author but restrictions apply to the availability of these data, which were used under license for the current study, and so are not publicly available. Data are however available with permission of the corresponding author.

\section{Ethics approval and consent to participate}

The study was conducted in accordance with the Helsinki Declaration. Informed consent was obtained from the patients. The Institutional Review Board of Qingdao Municipal Hospital approved the study.
Consent for publication

Not applicable.

\section{Competing interests}

The authors declared no potential conflicts of interest with respect to the research, authorship, and/or publication of this article.

\section{Author details}

'Department of Neurology, Qingdao Municipal Hospital, Qingdao University, Qingdao, China. ${ }^{2}$ Department of Anesthesiology, Qingdao Municipal Hospital, Qingdao University, Qingdao, China. ${ }^{3}$ Department of Neurology and Institute of Neurology, WHO Collaborating Center for Research and Training in Neurosciences, Huashan Hospital, Shanghai Medical College, Fudan University, 12th Wulumuqi Zhong Road, Shanghai 200040, China.

Received: 14 January 2020 Accepted: 2 April 2020

Published online: 10 April 2020

\section{References}

1. Eikelenboom P, Veerhuis R, van Exel E, Hoozemans JJ, Rozemuller AJ, van Gool WA. The early involvement of the innate immunity in the pathogenesis of late-onset Alzheimer's disease: neuropathological, epidemiological and genetic evidence. Curr Alzheimer Res. 2011;8(2):142-50.

2. Heneka MT, Golenbock DT, Latz E. Innate immunity in Alzheimer's disease. Nat Immunol. 2015:16(3):229-36.

3. Hickman SE, Kingery ND, Ohsumi TK, Borowsky ML, Wang LC, Means TK, et al. The microglial sensome revealed by direct RNA sequencing. Nat Neurosci. 2013:16(12):1896-905.

4. Kleinberger $G$, Yamanishi $Y$, Suarez-Calvet M, Czirr E, Lohmann E, Cuyvers $E_{\text {, }}$ et al. TREM2 mutations implicated in neurodegeneration impair cell surface transport and phagocytosis. Sci Transl Med. 2014;6(243):243ra86.

5. Colonna M, Wang Y. TREM2 variants: new keys to decipher Alzheimer disease pathogenesis. Nat Rev Neurosci. 2016;17(4):201-7.

6. Benitez BA, Cruchaga C. TREM2 and neurodegenerative disease. N Engl J Med. 2013;369(16):1567-8.

7. Guerreiro R, Wojtas A, Bras J, Carrasquillo M, Rogaeva E, Majounie E, et al. TREM2 variants in Alzheimer's disease. N Engl J Med. 2013;368(2):117-27.

8. Wunderlich P, Glebov K, Kemmerling N, Tien NT, Neumann H, Walter J. Sequential proteolytic processing of the triggering receptor expressed on myeloid cells-2 (TREM2) protein by ectodomain shedding and gammasecretase-dependent intramembranous cleavage. J Biol Chem. 2013;288(46): 33027-36.

9. Piccio L, Buonsanti C, Cella M, Tassi I, Schmidt RE, Fenoglio C, et al. Identification of soluble TREM-2 in the cerebrospinal fluid and its association with multiple sclerosis and CNS inflammation. Brain. 2008;131(Pt 11):3081-91.

10. Heslegrave A, Heywood W, Paterson R, Magdalinou N, Svensson J, Johansson P, et al. Increased cerebrospinal fluid soluble TREM2 concentration in Alzheimer's disease. Mol Neurodegener. 2016;11:3.

11. Piccio L, Deming Y, Del-Aguila JL, Ghezzi L, Holtzman DM, Fagan AM, et al. Cerebrospinal fluid soluble TREM2 is higher in Alzheimer disease and associated with mutation status. Acta Neuropathol. 2016;131(6):925-33.

12. Suarez-Calvet $M$, Kleinberger $G$, Araque Caballero MA, Brendel $M$, Rominger A, Alcolea D, et al. sTREM2 cerebrospinal fluid levels are a potential biomarker for microglia activity in early-stage Alzheimer's disease and associate with neuronal injury markers. EMBO Mol Med. 2016;8(5):466-76.

13. Soldan A, Pettigrew C, Cai Q, Wang MC, Moghekar AR, O'Brien RJ, et al. Hypothetical preclinical Alzheimer disease groups and longitudinal cognitive change. JAMA Neurol. 2016:73(6):698-705.

14. Sperling RA, Aisen PS, Beckett LA, Bennett DA, Craft S, Fagan AM, et al. Toward defining the preclinical stages of Alzheimer's disease: recommendations from the National Institute on Aging-Alzheimer's Association workgroups on diagnostic guidelines for Alzheimer's disease. Alzheimer's Dementia. 2011;7(3):280-92.

15. Blennow K, Hampel H, Weiner M, Zetterberg H. Cerebrospinal fluid and plasma biomarkers in Alzheimer disease. Nat Rev Neurol. 2010;6(3):131-44.

16. Desikan RS, Cabral HJ, Hess CP, Dillon WP, Glastonbury CM, Weiner MW et al. Automated MRI measures identify individuals with mild cognitive impairment and Alzheimer's disease. Brain. 2009;132(Pt 8):2048-57.

17. Grothe MJ, Heinsen H, Amaro E Jr, Grinberg LT, Teipel SJ. Cognitive Correlates of Basal Forebrain Atrophy and Associated Cortical 
Hypometabolism in Mild Cognitive Impairment. Cereb Cortex. 2016;26(6): 2411-26.

18. Morris JC, Roe CM, Xiong C, Fagan AM, Goate AM, Holtzman DM, et al. APOE predicts amyloid-beta but not tau Alzheimer pathology in cognitively normal aging. Ann Neurol. 2010;67(1):122-31.

19. Reiman EM, Chen K, Liu X, Bandy D, Yu M, Lee W, et al. Fibrillar amyloidbeta burden in cognitively normal people at 3 levels of genetic risk for Alzheimer's disease. Proc Natl Acad Sci U S A. 2009;106(16):6820-5.

20. Rowe CC, Ellis KA, Rimajova M, Bourgeat P, Pike KE, Jones G, et al. Amyloid imaging results from the Australian imaging, biomarkers and lifestyle (AIBL) study of aging. Neurobiol Aging. 2010;31(8):1275-83.

21. Bennett DA, Schneider JA, Arvanitakis Z, Kelly JF, Aggarwal NT, Shah RC, et al. Neuropathology of older persons without cognitive impairment from two community-based studies. Neurology. 2006;66(12):1837-44.

22. Hulette CM, Welsh-Bohmer KA, Murray MG, Saunders AM, Mash DC, McIntyre LM. Neuropathological and neuropsychological changes in "normal" aging: evidence for preclinical Alzheimer disease in cognitively normal individuals. J Neuropathol Exp Neurol. 1998;57(12):1168-74.

23. Hong YJ, Park KW, Kang DY, Lee JH. Prediction of Alzheimer's pathological changes in subjective cognitive decline using the self-report questionnaire and neuroimaging biomarkers. Dementia Neurocognitive Disord. 2019;18(1):19-29.

24. Hwang J, Jeong JH, Yoon SJ, Park KW, Kim EJ, Yoon B, et al. Clinical and Biomarker Characteristics According to Clinical Spectrum of Alzheimer's Disease (AD) in the Validation Cohort of Korean Brain Aging Study for the Early Diagnosis and Prediction of AD. J Clin Med. 2019;8:3.

25. Jang H, Kim HJ, Park S, Park YH, Choe Y, Cho H, et al. Application of an amyloid and tau classification system in subcortical vascular cognitive impairment patients. Eur J Nucl Med Mol Imaging. 2020;47(2):292-303.

26. Jack CR Jr, Knopman DS, Weigand SD, Wiste HJ, Vemuri P, Lowe V, et al. An operational approach to National Institute on Aging-Alzheimer's Association criteria for preclinical Alzheimer disease. Ann Neurol. 2012;71(6):765-75.

27. Albert MS, DeKosky ST, Dickson D, Dubois B, Feldman HH, Fox NC, et al. The diagnosis of mild cognitive impairment due to Alzheimer's disease: recommendations from the National Institute on Aging-Alzheimer's Association workgroups on diagnostic guidelines for Alzheimer's disease. Alzheimer's Dementia. 2011;7(3):270-9.

28. McKhann GM, Knopman DS, Chertkow H, Hyman BT, Jack CR Jr, Kawas CH, et al. The diagnosis of dementia due to Alzheimer's disease: recommendations from the National Institute on Aging-Alzheimer's Association workgroups on diagnostic guidelines for Alzheimer's disease. Alzheimer's Dementia. 2011;7(3):263-9.

29. Schott JM, Revesz T. Inflammation in Alzheimer's disease: insights from immunotherapy. Brain. 2013;136(Pt 9):2654-6.

30. Rauchmann BS, Schneider-Axmann T, Alexopoulos P, Perneczky R. CSF soluble TREM2 as a measure of immune response along the Alzheimer's disease continuum. Neurobiol Aging. 2019;74:182-90.

31. Wang Y, Cella M, Mallinson K, Ulrich JD, Young KL, Robinette ML, et al. TREM2 lipid sensing sustains the microglial response in an Alzheimer's disease model. Cell. 2015;160(6):1061-71.

32. Suarez-Calvet M, Morenas-Rodriguez E, Kleinberger G, Schlepckow K, Araque Caballero MA, Franzmeier N, et al. Early increase of CSF sTREM2 in Alzheimer's disease is associated with tau related-neurodegeneration but not with amyloid-beta pathology. Mol Neurodegener. 2019;14(1):1.

33. Condello C, Yuan P, Schain A, Grutzendler J. Microglia constitute a barrier that prevents neurotoxic protofibrillar Abeta42 hotspots around plaques. Nat Commun. 2015;6:6176.

34. Wang Y, Ulland TK, Ulrich JD, Song W, Tzaferis JA, Hole JT, et al. TREMZmediated early microglial response limits diffusion and toxicity of amyloid plaques. J Exp Med. 2016;213(5):667-75.

35. Parhizkar S, Arzberger T, Brendel M, Kleinberger G, Deussing M, Focke C, et al. Loss of TREM2 function increases amyloid seeding but reduces plaque-associated ApoE. Nat Neurosci. 2019;22(2):191-204.

36. Frank S, Burbach GJ, Bonin M, Walter M, Streit W, Bechmann I, et al. TREM2 is upregulated in amyloid plaque-associated microglia in aged APP23 transgenic mice. Glia. 2008;56(13):1438-47.

37. Lue LF, Schmitz CT, Serrano G, Sue LI, Beach TG, Walker DG. TREM2 Protein Expression Changes Correlate with Alzheimer's Disease Neurodegenerative Pathologies in Post-Mortem Temporal Cortices. Brain Pathol (Zurich, Switzerland). 2015;25(4):469-80.

38. Holtman IR, Raj DD, Miller JA, Schaafsma W, Yin Z, Brouwer N, et al. Induction of a common microglia gene expression signature by aging and neurodegenerative conditions: a co-expression meta-analysis. Acta Neuropathol Commun. 2015;3:31.

39. Keren-Shaul H, Spinrad A, Weiner A, Matcovitch-Natan O, Dvir-Szternfeld R, Ulland TK, et al. A Unique Microglia Type Associated with Restricting Development of Alzheimer's Disease. Cell. 2017;169(7):1276-90.e17.

40. Krasemann S, Madore C, Cialic R, Baufeld C, Calcagno N, El Fatimy R, et al. The TREM2-APOE Pathway Drives the Transcriptional Phenotype of Dysfunctional Microglia in Neurodegenerative Diseases. Immunity. 2017; 47(3):566-81.e9.

41. Paternico D, Galluzzi S, Drago V, Bocchio-Chiavetto L, Zanardini R, Pedrini L, et al. Cerebrospinal fluid markers for Alzheimer's disease in a cognitively healthy cohort of young and old adults. Alzheimer's Dementia. 2012;8(6): 520-7.

42. Sjogren M, Vanderstichele H, Agren H, Zachrisson O, Edsbagge M, Wikkelso C, et al. Tau and Abeta42 in cerebrospinal fluid from healthy adults 21-93 years of age: establishment of reference values. Clin Chem. 2001;47(10): 1776-81.

43. Sato C, Barthelemy NR, Mawuenyega KG, Patterson BW, Gordon BA, JockelBalsarotti J, et al. Tau Kinetics in Neurons and the Human Central Nervous System. Neuron. 2018;97(6):1284-98.e7.

44. Gispert JD, Suarez-Calvet M, Monte GC, Tucholka A, Falcon C, Rojas S, et al. Cerebrospinal fluid sTREM2 levels are associated with gray matter volume increases and reduced diffusivity in early Alzheimer's disease. Alzheimer's Dementia. 2016;12(12):1259-72.

45. Jonsson T, Stefansson H, Steinberg S, Jonsdottir I, Jonsson PV, Snaedal J, et al. Variant of TREM2 associated with the risk of Alzheimer's disease. N Engl J Med. 2013;368(2):107-16.

\section{Publisher's Note}

Springer Nature remains neutral with regard to jurisdictional claims in published maps and institutional affiliations.
Ready to submit your research? Choose BMC and benefit from:

- fast, convenient online submission

- thorough peer review by experienced researchers in your field

- rapid publication on acceptance

- support for research data, including large and complex data types

- gold Open Access which fosters wider collaboration and increased citations

- maximum visibility for your research: over $100 \mathrm{M}$ website views per year

At BMC, research is always in progress.

Learn more biomedcentral.com/submissions 